acupuncture therapy is the virtual absence of life-threatening side effects and complications resulting from its use. ${ }^{3}$ However, it would be expected that life-threatening cardiovascular complications like those in this report (i.e., cardiac tamponade) could result from the use of acupuncture needles. A previous report ${ }^{4}$ described a serious complication as a consequence of applying needles to the thorax directly, which caused cardiac injury and tamponade. Several pathways by which the acupuncture needle could have reached the heart can be considered, ${ }^{2}$ but we believe that in my patient the needle reached the right ventricle via the bloodstream from a distant region, eventually penetrating the free wall, because of the location of the acupuncture therapy and the coexistence of several needles in the pulmonary vasculature. In patients such as this, surgical removal of the pins or needles in the heart should be attempted because of the high mortality rate of needle-related cardiac injury. ${ }^{2}$ To facilitate successful removal of a needle from the heart, it is necessary to define the precise location of the needle before or during the operation. Ultrasonography has been reported to be useful for the evaluation and management of patients with foreign bodies in the heart, ${ }^{5}$ although I failed to find the cardiac needle before operating using this method. Instead, $\mathrm{x}$-ray computed tomography with contrast medium enabled me to locate the needle in the heart and greatly facilitated its successful removal, although the image was accompanied by an object-related artifact.
In conclusion, physicians involved in acupuncture therapy should keep in mind the possible serious cardiac complications reported here. Contrast x-ray computed tomography may be useful for imaging the needle in the heart and great vessels.

I thank Drs. Yoshiaki Mori and Tetsuo Hadama of the Department of Cardiovascular Surgery at the Oita Medical University for their help in treating the patient.

\section{REFERENCES}

1. Shiraishi S, Goto I, Kuroiwa Y, Nishio S, Kinoshita K. Spinal cord injury as a complication of acupuncture. Neurology 1979;29:1188-90.

2. Schechter DC, Gilbert L. Injuries of the heart and great vessels due to pins and needles. Thorax 1969;24:246-53.

3. Rapson LM. Acupuncture: a useful treatment modality. Acupunc Res Q 1984;8:15-27.

4. Rosted P. Literature survey of reported adverse effects associated with acupuncture treatment. Am J Acupunc 1996;24: $27-34$.

5. Harrison LH Jr, Kisslo JA Jr, Sabiston DC Jr. Extraction of intramyocardial foreign body utilizing operative ultrasonography. J Thorac Cardiovase Surg 1981;82:345-9.

\title{
LONG-TERM FOLLOW-UP OF APROTININ-SPECIFIC IMMUNOGLOBULIN G ANTIBODIES AFTER CARDIAC OPERATIONS
}

\author{
J. Weipert, MD, ${ }^{a}$ H. Meisner, MD, ${ }^{a}$ M. Jochum, $\mathrm{PhD},{ }^{b}$ and W. Dietrich, MD, ${ }^{c}$ Munich, Germany
}

The protease inhibitor aprotinin (Trasylol, Bayer AG, Leverkusen, Germany) is used in cardiac operations for acquired heart disease to reduce postoperative bleeding and allogeneic blood requirement. ${ }^{1}$ Aprotinin is a polybasic polypeptide derived from bovine lung, and antibody production in human beings is likely to occur. Recently,

From the Clinic of Heart and Vascular Surgery, German Heart

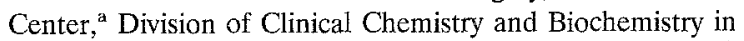
the Department of Surgery, Ludwig-Maximilians-University, ${ }^{b}$ and Institute of Anesthesiology, German Heart Center, Technical University of Munich, Munich, Germany.

Received for publication Dec. 23, 1996; accepted for publication March 12, 1997.

Address for reprints: Joachim Weipert, MD, Klinik für Herz- und Gefä $\beta$ chirurgie, Deutsches Herzzentrum München, Lazarettstr. 36, 80636 München, Germany.

J Thorac Cardiovasc Surg 1997;114:676-8

Copyright $\mathbb{0} 1997$ by Mosby-Year Book, Inc.

$0022-5223 / 97 \$ 5.00+0 \quad \mathbf{1 2 / 5 4 / 8 1 9 4 8}$ deaths resulting from anaphylactic reactions after systemic aprotinin exposure in cardiac operations have been reported. ${ }^{2}$ However, it is unknown by which pathway these reactions are mediated. Because the use of aprotinin at initial cardiac operations is widespread, at least in Europe, the risk for anaphylactic reactions at aprotinin reexposure may be increased. To study parameters to assess patients' individual risks for adverse reactions to aprotinin, we followed the course of aprotinin-specific antibodies of the immunoglobulin (IG) $G$ class for 4 years.

With institutional approval and after informed consent, 36 consecutive adult patients having cardiac operations could be followed up. Only patients with no previous exposure to aprotinin were selected for this study. Blood specimens were drawn for the first time during their postoperative hospital stay or on an outpatient basis, but within 4 months after the operation. Four years later these patients were reinvestigated. Aprotinin was used according to the following protocol: A test dose of $10,000 \mathrm{KIU}$ of aprotinin $(1 \mathrm{ml})$ was given 10 minutes before the first bolus. Patients received an initial bolus of $2 \times 10^{6} \mathrm{KIU}$ of 
The Journal of Thoracic and

Cardiovascular Surgery

Brief communications

677

Volume 114, Number 4

Table I. Semiquantitative assessment of aprotinin-specific $\operatorname{Ig} G$ antibodies in 36 patients

\begin{tabular}{|c|c|c|c|}
\hline \multirow{2}{*}{\multicolumn{4}{|c|}{ A. Overall ELISA test results }} \\
\hline & & & \\
\hline \multicolumn{4}{|l|}{$\begin{array}{l}\text { A. Overall ELISA test results } \\
\text { Median (range) time after exposure }\end{array}$} \\
\hline Positive for IgG antibodies & & $17(47.2 \%)$ & $14(38.9 \%)$ \\
\hline Questionably positive & & $7(19.4 \%)$ & $10(27.7 \%)$ \\
\hline Negative & & $12(33.4 \%)$ & $12(33.4 \%)$ \\
\hline \multicolumn{4}{|c|}{ B. IgG status at each ELISA test from immediately to 4 years after the operation } \\
\hline Change $(n)$ : & To positive & To questionable & To negative \\
\hline From negative & 3 & 3 & 6 \\
\hline From positive & 8 & 6 & 3 \\
\hline From questionable & 3 & 1 & 3 \\
\hline
\end{tabular}

aprotinin with the start of the operation, followed by an infusion of $5 \times 10^{5} \mathrm{KIU} / \mathrm{hr}$. Additionally, $2 \times 10^{6} \mathrm{KIU}$ of aprotinin was added to the pump prime.

By means of a semiquantitative, indirect enzyme-linked immunoassay (ELISA) test, the course of aprotinin-specific IgG antibodies was studied. In brief, ELISA plates were coated with aprotinin and incubated during 24 hours at $4^{\circ} \mathrm{C}$. The plates were washed thereafter. Test specimens were serially diluted $\left(1: 2^{\mathrm{n}}\right)$ and added to the wells. A positive sample with a serial dilution of 1:10 to 1:1200 was used to establish a semiquantitative standard curve. After an incubation time of 2 hours at $37^{\circ} \mathrm{C}$, the plates were washed again to remove unbound material. Then peroxidase-conjugated goat antihuman IgG antibodies were added and incubated for 3 hours at $37^{\circ} \mathrm{C}$. After additional washings, substrate reaction was achieved with $2,2^{\prime}$-azinobis(3-ethylbenzthiazoline-6-sulfonic acid) (ABTS) at $37^{\circ} \mathrm{C}$ and the absorbance was measured after 30 minutes at $405 \mathrm{~nm}$. The amount of aprotinin antibodies was directly proportional to the colored product. Specific IgG antibody titers below a sample dilution of $1: 20$ were considered as being negative, between $1: 20$ to $1: 50$ as being questionable, and above 1:100 as certainly positive. Data are presented as mean \pm standard deviation.

Twelve women and 24 men (median age 56 years, ranging from 33 to 67 years; coronary artery bypass grafting and valve operations) were studied. The final amount of aprotinin during extracorporeal circulation was $5.5 \pm 0.79 \times 10^{6} \mathrm{KIU}$. The clinical course of all patients was uneventful, and patients were not reexposed to aprotinin. After a median time of 7 days (ranging from 6 to 122 days) after the operation, 17 patients had positive test results and 7 patients had questionably positive test results for IgG antibodies (Table I, $A$ ). At that time 12 patients had negative test results for $\operatorname{IgG}$ antibodies. After a median of 48 months (ranging from 44 to 51 months) after aprotinin exposure, 14 patients had positive blood samples and 10 patients had questionably positive blood samples for IgG antibodies. In 12 patients no IgG antibodies could be found. Also, an intraindividual change of the aprotininspecific IgG antibody status from the first to the second investigation period could be observed (Table I, $B$ ). All patients with a change of the aprotinin-specific IgG status from negative to positive or questionably positive were tested for the first time within 6 to 9 days after aprotinin exposure. At both investigation periods, six (16.7\%) patients had negative test results for aprotinin-specific IgG antibodies.
Comment. Aprotinin, a polybasic polypeptide, is a naturally occurring inhibitor of proteolytic enzymes and is derived from bovine lungs. The blood-saving effect after aprotinin application during cardiac operations with extracorporal circulation is well established. ${ }^{1}$ As a xenogeneic protein it possesses antigenic properties, and for the cardiac surgical field the incidence of reported anaphylactic reactions at primary aprotinin exposition is between $0.5 \%$ and $1 \% .^{3}$ Because of the increasing rate of reoperations in patients having heart surgery, reliable parameters for measuring the patient's individual risk for adverse reaction to aprotinin reexposure are desirable. Up to now it is unclear which parameter or test systems are able to predict the risk of allergic aprotinin reaction. To our knowledge, the multicenter study by Ruskowski and colleagues ${ }^{4}$ involving 289 patients is the largest study so far. This study evaluated the immunologic sequel of aprotinin exposure up to 6 months after exposure in patients having cardiac operations. At that time the subcutaneous Prick test for aprotinin was positive in two patients only. The authors concluded that preoperative Prick testing at planned aprotinin reexposure might not be sensitive enough to predict the patient's risk of adverse aprotinin reaction. Aprotinin-specific IgG antibodies were found in $46 \%$ of the patients. However, the contribution for aprotinin-specific $\operatorname{IgG}$ antibodies to an allergic reaction to aprotinin is not yet established. ${ }^{4}$

During the early period after aprotinin exposure, the percentage of patients (47\%) with aprotinin-specific IgG antibodies in our study was nearly identical to the percentage reported by Ruskowski and coworkers. ${ }^{4}$ As early as 7 days after aprotinin exposure, positive test results could be found. Four years after aprotinin exposure, all intraindividual shifts of the $\operatorname{IgG}$ test results from positive to negative and vice versa were found (Table $I, B$ ). Although we did not reexpose the patients to aprotinin at the second evaluation, one might speculate that patients with positive IgG status are still at risk for allergic reaction to aprotinin reexposure. This theory is supported by our clinical data. The overall risk for adverse reaction to aprotinin reexposure was 7 of 248 reexposures (2.8\%) and the risk for adverse reaction was significantly higher within 4 months after aprotinin exposure as compared with late aprotinin reexposure $(6.4 \%$ vs $1.9 \%){ }^{3}$

Because of the documented blood-saving effect with aprotinin application in cardiac operations, reliable test systems are becoming an important issue of the comprehensive management of patients with reexposure to apro- 
tinin. We found that $47 \%$ and $39 \%$ of the patients had positive test results for aprotinin-specific $\operatorname{IgG}$ antibodies early and late after exposition. Not much is known about the precise immunologic sequel during an adverse reaction to aprotinin. Determination of aprotinin-specific $\operatorname{IgG}$ antibodies might be a useful clinical indicator to identify individuals at risk for anaphylactic reactions to aprotinin, but more precise methods are desirable.

\section{REFERENCES}

1. Lemmer JH, Stanford W, Bonney SL, Breen JF, Chomka EV, Eldredge WJ, et al. Aprotinin for coronary bypass operations: efficacy, safety, and influence on early saphenous vein graft patency - a multicenter, randomized, double-blind, placebocontrolled study. J Thorac Cardiovasc Surg 1994;107:543-51.

2. Diefenbach CM, Limpers AB, Lynch J, Ruskowski H, Jugert FK, Buzello W. Fatal anaphylactic shock after aprotinin reexposure in cardiac surgery. Anesth Analg 1995;80:830-1.

3. Dietrich W, Spaeth P, Ebell A, Richter JA. Incidence of anaphylactic reactions to aprotinin: analysis of 248 reexposures to aprotinin. J Thorac Cardiovasc Surg 1997;113:194-201.

4. Ruskowski H, Joos A, Kiefer H, Soeparwata R, Wendt G, Merk $\mathrm{H}$, et al. Untersuchungen zur Antigenität von Trasylol in der offenen Herzchirurgie. J Thorac Cardiovasc Surg 1993;41:86.

\section{PRIMARY TRACHEAL SYNOVIAL CELL SARCOMA: A FIRST CASE REPORT}

Anne T. Sykes, MD, ${ }^{a}$ Chris K. Rokkas, MD, ${ }^{a}$ Andre Kajdacsy-Balla, MD, ${ }^{b}$ and George B. Haasler, MD, ${ }^{a}$ Milwaukee, Wis.

Synovial cell sarcoma is a rare soft-tissue tumor comprising only $7 \%$ of all soft-tissue sarcomas, with fewer than $10 \%$ of those involving the head and neck. It has never been described involving the trachea as the primary site. We report here a case of primary tracheal synovial cell sarcoma and review the limited data available on diagnosis, prognosis, and treatment of this tumor.

Clinical summary. A 20-year-old white male college student with a 3-year history of exercise-induced asthma was being treated with bronchodilators. Progressive dyspnea, stridor, intermittent hemoptysis, and a $15 \mathrm{~kg}$ weight loss developed over a 3-month period. Coughing episodes elicited anterior chest pain radiating to the back. An episode of respiratory distress prompted a visit to the emergency department. Results of physical examination were remarkable for respiratory stridor and wheezing. The patient continued to have symptoms despite systemic steroid therapy. Bronchoscopy revealed a smooth, pale, soft tracheal tumor extending proximally to $3 \mathrm{~cm}$ below the true vocal cords, occupying most of the tracheal lumen. Computed tomography demonstrated a $3 \mathrm{~cm}$ intratracheal mass extending outside the tracheal wall, with its distal extension $5 \mathrm{~cm}$ above the carina (Fig. 1).

From the Departments of Cardiothoracic Surgery a and Pathology, ${ }^{\mathrm{b}}$ Medical College of Wisconsin, Milwaukee, Wis.

Received for publication Oct. 28, 1996; accepted for publication Jan. 8, 1997.

Address for reprints: George B. Haasler, MD, Medical College of Wisconsin, Department of Cardiothoracic Surgery, $9200 \mathrm{~W}$. Wisconsin Ave., Milwaukee, WI 53226-0099.

J Thorac Cardiovasc Surg 1997;114:678-80

Copyright $(1997$ by Mosby-Year Book, Inc.

$0022-5223 / 97 \$ 5.00+0 \quad \mathbf{1 2 / 5 4 / 8 0 2 3 3}$
There was neither clinical nor radiographic evidence of lymphadenopathy.

The patient underwent en bloc tumor excision with tracheal resection by a neck approach through a low collar incision. Intraoperative bronchoscopy revealed a normal distal tracheobronchial tree. Five tracheal rings with clear surgical margins of at least $1 \mathrm{~cm}$ were removed, and primary end-to-end anastomosis was performed. Grossly, the mass appeared well circumscribed, with transmural extension into the paratracheal region but without gross invasion into fat. The patient recovered uneventfully and was discharged to home on the fourth postoperative day without bronchodilators. Results of metastatic workup, including computed tomography of the chest, abdomen, and pelvis and a bone scan, were negative. $\mathrm{He}$ was referred to radiation oncology and received a total of 62 Gy external beam irradiation in 22 fractions of $180 \mathrm{cGy}$ and boost therapy in 12 fractions of $180 \mathrm{cGy}$. He has not been treated with adjuvant chemotherapy. The patient remains well 8 months after the operation.

Pathology. Histologically, the tumor showed biphasic growth. The pattern was composed mostly of ill-defined interweaving fascicles of monomorphic spindle cells but also showed numerous clear-cut foci of cuboidal cells forming pseudoglandular clusters (Fig. $2, a$ ). This biphasic pattern was even more obvious when immunohistochemical stains were used to show mutually exclusive vimentin-positive spindle cells and the epithelial component positive for low-molecular weight cytokeratin and carcinoembryonic antigen (Fig. 2,b). Numerous scattered mast cells were seen. Both components of the tumor stained negative for intracellular mucin (Alcian blue at pH 2.5, periodic acid-Schiff, and mucicarmine), argyrophilic granules (Grimelius stain), and immunohistochemically for chromogranin, S-100, B72.3, smooth-muscle actin, and desmin. 